\title{
Fano Effect through Parallel-coupled Double Coulomb Islands
}

\author{
Haizhou Lu, ${ }^{1, \text { * }}$ Rong Lü, ${ }^{1}$ and Bang-fen Zhu ${ }^{1,2,0}$ \\ ${ }^{1}$ Center for Advanced Study, Tsinghua University, Beijing 100084, China \\ ${ }^{2}$ Department of Physics, Tsinghua University, Beijing 100084, China
}

\begin{abstract}
By means of the non-equilibrium Green function and equation of motion method, the electronic transport is theoretically studied through a parallel-coupled double quantum dots(DQD) in the presence of the on-dot Coulomb correlation, with an emphasis put on the quantum interference. It has been found that in the Coulomb blockage regime, the quantum interference between the bonding and antiboding DQD states or that between their Coulomb blockade counterparts may result in the Fano resonance in the conductance spectra, and the Fano peak doublet may be observed under certain non-equilibrium condition. The possibility of manipulating the Fano lineshape is predicted by tuning the dot-lead coupling and magnetic flux threading the ring connecting the dots and leads. Similar to the case without Coulomb interaction, the direction of the asymmetric tail of Fano lineshape can be flipped by the external field. Most importantly, by tuning the magnetic flux, the function of four relevant states can be interchanged, giving rise to the swap effect, which might play a key role as a qubit in the quantum computation.

PACS numbers: 73.23.Hk, 73.63.Kv, 73.40.Gk
\end{abstract}

\section{INTRODUCTION}

Fano resonance stems from quantum interference between resonant and nonresonant processes, $\frac{1}{\stackrel{1}{2}}$ and manifests itself in spectra as asymmetric lineshape in a large variety of experiments. It is known that Fano effect is a good probe for the phase coherence for carriers in solids, in particular in a quantum dot (QD) system.2.3.4.5.6.7 Unlike the conventional Fano resonance, $\frac{8.9 .10 .11}{1}$ the Fano effect in the QD system has its advantage in that its key parameters can be readily tuned. Suppose a discrete level inside the QD is broadened by a factor of $\Gamma$ due to coupling to the continua in leads, the key to realize the Fano effect in the conductance spectra is that within $\Gamma$ centered at the resonance level, the phase of the electron in the non-resonant channel changes little 12.13 The first observation of the Fano lineshape in the QD system was reported by Göres et al $l^{3.4}$ in the single-electrontransistor experiments. Recently, K. Kobayashi et al. studied the magnetically and electrostatically tuned Fano effect in a QD embedded in an Aharonov-Bohm(AB) ring, $\stackrel{5.6}{5}$ and A.C. Johnson et al. investigated a tunable Fano interferometer consisting of a QD coupled to a one-dimensional channel via tunneling and observed the Coulomb-modified Fano resonances $\underline{\underline{\underline{z}}}$

Recent experimental advances in the parallel-coupled double quantum dots (DQD) 14.15.16.17.18, in which two coupled QD's via barrier tunneling are embedded into opposite arms of a $\mathrm{AB}$ ring respectively and also coupled to two leads roughly equally ( $c f$ Fig 1), have inspired a number of theoretical attempts to study the coherent transport in this system. $19,20,21,22.23$ As a controllable two-level system, it is appealing for the parallel-coupled DQD system to become one of promising candidates for the quantum bit in quantum computation based on solid state devices ${ }^{24}$ The entangled quantum states required for performing the quantum computation demand a high degree of phase coherence in the system. ${ }^{25}$ Being a probe to the phase coherence, $\stackrel{26}{2}$ the Fano effect in the parallelcoupled DQD system is certainly of practical importance, if tunable, and especially if the swap effect can be manipulated.

In a real small system like quantum dots, the electronelectron (e-e) interaction will influence the transport process. A question naturally arises, whether the e-e interaction in the QD breaks the Fano resonance in the DQD system or not? Till now, most theoretical works addressing the Fano effect in DQD ignored this aspect by adopting a Fano-Anderson model in which the e-e repulsion is entirely absent 19.20 .21 .22 .23 The electron correlations in the DQD have been taken into account in coherent electronic transport through a DQD connected in a series

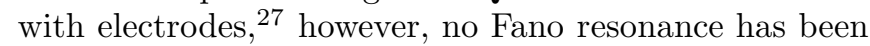
found in this configuration. In this article, we intend to investigate the Fano effect in the tunneling-coupled parallel DQD system in the presence of finite on-dot Coulomb interactions, that is, the Fano effect in Parallel-coupled Double Coulomb Islands.

The paper is organized as follows. After introducing the two-impurity Anderson model with an inter-dot coupling term to describe the parallel-coupled DQD system in Sec III the current through the mesoscopic system is formulated in Sec III with the Green functions in the central region and Fermi distribution in leads ${ }^{28}$ In small $U$ cases, all spin flip processes may be ignored, within the Hatree-Fock approximation(HFA), the current formula is reduced to a Landauer-Büttiker one, and the electrons with spin $\sigma$ behave like moving in a mean field of electrons with the opposite spin $\bar{\sigma}$. Then numerical results for the zero temperature case are presented in Sec IV Owing to the on-dot repulsion and inter-dot coupling, there are four quantum states in the DQD system relevant, centered approximately at $\varepsilon_{0}-t_{c}, \varepsilon_{0}+t_{c}, \varepsilon_{0}-t_{c}+U$ and $\varepsilon_{0}+t_{c}+U$, corresponding to the bonding and antibonding DQD states and their Coulomb blockade counterparts, respectively. It has been found that the Fano 
resonance appears as a result of the quantum interference not only between the DQD states at $\varepsilon_{0}-t_{c}$ and $\varepsilon_{0}+t_{c}$, but also between their Coulomb blockade counterparts. It has also been found that the direction of the asymmetric tail of Fano lineshape can be flipped by the external field. Most importantly, by tuning the total magnetic flux through the AB ring, the swap effect between four resonance peaks in the conductance spectra is predicted, which might be of potential application as a type of CNot gate in the quantum computation. Finally, a brief summary is drawn and presented.

\section{PHYSICAL MODEL}

The total Hamiltonian is expressed as

$$
\mathrm{H}=\mathrm{H}_{\text {leads }}+\mathrm{H}_{D Q D}+\mathrm{H}_{T} \text {. }
$$

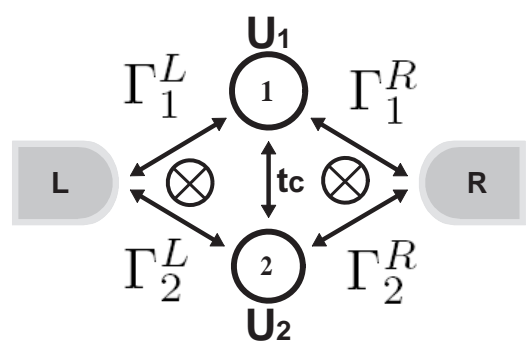

FIG. 1: Schematic setup of a tunneling-coupled parallel DQD system coupled to two reservoirs.

We start with the two-impurity Anderson model for the parallel-coupled DQD ( $c f$ Fig 1) as

$$
\begin{aligned}
\mathrm{H}_{D Q D} & =\sum_{i, \sigma} \varepsilon_{i \sigma} d_{i \sigma}^{\dagger} d_{i \sigma}+\sum_{i} U_{i} d_{i \uparrow}^{\dagger} d_{i \uparrow} d_{i \downarrow}^{\dagger} d_{i \downarrow} \\
& -t_{c} \sum_{\sigma}\left(d_{1 \sigma}^{\dagger} d_{2 \sigma}+\text { h.c. }\right)
\end{aligned}
$$

where $d_{i \sigma}^{\dagger}\left(d_{i \sigma}\right)$ represents the creation (annihilation) operator for the discrete state with the energy $\varepsilon_{i \sigma}$ and spin $\sigma(\sigma=\uparrow, \downarrow)$ in $\operatorname{dot} i(i=1,2)$, which are coupled each other via tunneling $t_{c}$, and the on-dot Coulomb repulsion is described by the second term in Eq.(2).

The $\mathrm{H}_{\text {leads }}$ in Eq. (1) represents the noninteracting electron gas in the left $(\mathrm{L})$ and right $(\mathrm{R})$ leads,

$$
\mathrm{H}_{l e a d s}=\sum_{k, \alpha, \sigma} \varepsilon_{k \alpha} c_{k \alpha \sigma}^{\dagger} c_{k \alpha \sigma},
$$

where $c_{k \alpha \sigma}^{\dagger}\left(c_{k \alpha \sigma}\right)$ is the creation (annihilation) operator for a continuous state in the lead $\alpha(\alpha=L, R)$ with energy $\varepsilon_{k \alpha}$ and spin $\sigma$.

The $\mathrm{H}_{T}$ in Eq.(1) represents the tunneling coupling between the QD and lead electrons,

$$
\mathrm{H}_{T}=\sum_{k, \alpha, \sigma, i} V_{\alpha i} d_{i \sigma}^{\dagger} c_{k \alpha \sigma}+\text { h.c. }
$$

where for the sake of simplicity the tunneling matrix element $V_{\alpha i}$ is assumed to be independent of $k$, and the phase shift due to the total magnetic flux threading into the $\mathrm{AB}$ ring, $\Phi$, is assumed to distribute evenly among 4 sections of the DQD-AB ring, namely $V_{L 1}=$ $\left|V_{L 1}\right| e^{i \frac{\phi}{4}}, V_{L 2}^{*}=\left|V_{L 2}\right| e^{i \frac{\phi}{4}}, V_{R 1}^{*}=\left|V_{R 1}\right| e^{i \frac{\phi}{4}}$, and $V_{R 2}=$ $\left|V_{R 2}\right| e^{i \frac{\phi}{4}}$. Here $\phi=2 \pi \Phi / \Phi_{0}$, in which the flux quantum $\Phi_{0}=h c / e$. In the following calculation, we define the line-width matrix as $\Gamma_{i j}^{\alpha} \equiv \sum_{k} V_{\alpha i} V_{\alpha j}^{*} 2 \pi \delta\left(\varepsilon-\varepsilon_{k \alpha}\right)$ $(\alpha=L, R)$ and $\boldsymbol{\Gamma}=\boldsymbol{\Gamma}^{L}+\boldsymbol{\Gamma}^{R}$. According to Fig 1 the line-width matrices in the $\mathrm{QD}$ representation read

$$
\boldsymbol{\Gamma}^{L}=\left(\begin{array}{cc}
\Gamma_{1}^{L} & \sqrt{\Gamma_{1}^{L} \Gamma_{2}^{L}} e^{i \frac{\phi}{2}} \\
\sqrt{\Gamma_{1}^{L} \Gamma_{2}^{L}} e^{-i \frac{\phi}{2}} & \Gamma_{2}^{L}
\end{array}\right),
$$

and

$$
\Gamma^{R}=\left(\begin{array}{cc}
\Gamma_{1}^{R} & \sqrt{\Gamma_{1}^{R} \Gamma_{2}^{R}} e^{-i \frac{\phi}{2}} \\
\sqrt{\Gamma_{1}^{R} \Gamma_{2}^{R}} e^{i \frac{\phi}{2}} & \Gamma_{2}^{R}
\end{array}\right),
$$

where $\Gamma_{i}^{\alpha}$ is short for $\Gamma_{i i}^{\alpha}$.

\section{CURRENT FORMULA}

According to Meir and Wingreen, 28 the general formula for current through a mesoscopic region between noninteracting leads is given by

$$
\begin{aligned}
J= & \sum_{\sigma} \frac{i e}{2 h} \int d \omega \operatorname{Tr}\left\{\left(\boldsymbol{\Gamma}^{L}-\boldsymbol{\Gamma}^{R}\right) \mathbf{G}^{<}(\omega)\right. \\
& \left.+\left[f_{L}(\omega) \boldsymbol{\Gamma}^{L}-f_{R}(\omega) \boldsymbol{\Gamma}^{R}\right]\left(\mathbf{G}^{r}(\omega)-\mathbf{G}^{a}(\omega)\right)\right\}
\end{aligned}
$$

where, $f_{L(R)}(\omega)$ is the Fermi distribution function on the left(right) leads, $\boldsymbol{\Gamma}^{L(R)}$ has been given by Eq.(5), $\mathbf{G}^{r}$, $\mathbf{G}^{a}$ and $\mathbf{G}^{<}$are the retarded, advanced and lesser Green functions in the DQD region, respectively.

\section{A. Retarded and Advanced Green Functions}

The retarded Green function is defined as

$$
G_{i \sigma, j \sigma}^{r}(t) \equiv\left\langle\left\langle d_{i \sigma}(t) \mid d_{j \sigma}^{\dagger}\right\rangle\right\rangle^{r} \equiv-i \theta(t)\left\langle\left\{d_{i \sigma}(t), d_{j \sigma}^{\dagger}\right\}\right\rangle .
$$

Writing the equation of motion for the retarded Green function in Fourier space $\left\langle\left\langle d_{i \sigma} \mid d_{j \sigma}^{\dagger}\right\rangle\right\rangle_{\omega}^{r}{ }^{29}$ one arrives at

$$
\begin{aligned}
& \left(\omega-\varepsilon_{i \sigma}+\frac{i}{2} \Gamma_{i i}\right)\left\langle\left\langle d_{i \sigma} \mid d_{j \sigma}^{\dagger}\right\rangle\right\rangle_{\omega}^{r}+\left(t_{c}+\frac{i}{2} \Gamma_{i \bar{i}}\right)\left\langle\left\langle d_{\bar{i} \sigma} \mid d_{j \sigma}^{\dagger}\right\rangle\right\rangle_{\omega}^{r} \\
& =\delta_{i j}+U_{i}\left\langle\left\langle d_{i \sigma} n_{i \bar{\sigma}} \mid d_{j \sigma}^{\dagger}\right\rangle\right\rangle_{\omega}^{r},
\end{aligned}
$$

where, $\bar{i}=2$ if $i=1$, and vice versa. During the derivation, we have calculated

$$
\left\langle\left\langle c_{k \alpha \sigma} \mid d_{i \sigma}^{\dagger}\right\rangle\right\rangle_{\omega}^{r}=\sum_{j=1,2} \frac{V_{\alpha j}^{*}\left\langle\left\langle d_{j \sigma} \mid d_{i \sigma}^{\dagger}\right\rangle\right\rangle_{\omega}^{r}}{\omega-\varepsilon_{k \alpha}+i 0^{+}},
$$


and defined the retarded self energy originated from the dot-lead couplings as

$$
\Sigma_{i j}^{r}=\sum_{k, \alpha} \frac{V_{\alpha i} V_{\alpha j}^{*}}{\omega-\varepsilon_{k \alpha}+i 0^{+}},
$$

where $0^{+}$represents an infinitesimal. In the wide-band limit,

$$
\Sigma_{i j}^{r} \approx-\frac{i}{2}\left(\Gamma_{i j}^{L}+\Gamma_{i j}^{R}\right)=-\frac{i}{2} \Gamma_{i j} .
$$

The equation of motion for the last term in Eq. (8), $\left\langle\left\langle d_{i \sigma} n_{i \bar{\sigma}} \mid d_{j \sigma}^{\dagger}\right\rangle\right\rangle_{\omega}^{r}$, reads

$$
\begin{aligned}
& \left(\omega-\varepsilon_{i \sigma}-U_{i}\right)\left\langle\left\langle d_{i \sigma} n_{i \bar{\sigma}} \mid d_{j \sigma}^{\dagger}\right\rangle\right\rangle_{\omega}^{r} \\
= & \delta_{i j}\left\langle n_{i \bar{\sigma}}\right\rangle-t_{c}\left\langle\left\langle d_{\bar{i} \sigma} n_{i \bar{\sigma}} \mid d_{j \sigma}^{\dagger}\right\rangle\right\rangle_{\omega}^{r}-t_{c}\left\langle\left\langle d_{i \sigma} d_{i \bar{\sigma}}^{\dagger} d_{\bar{i} \bar{\sigma}} \mid d_{j \sigma}^{\dagger}\right\rangle\right\rangle_{\omega}^{r} \\
+ & t_{c}\left\langle\left\langle d_{i \sigma} d_{\bar{i} \bar{\sigma}}^{\dagger} d_{i \bar{\sigma}} \mid d_{j \sigma}^{\dagger}\right\rangle\right\rangle_{\omega}^{r}+\sum_{k, \alpha} V_{\alpha i}\left\langle\left\langle c_{k \alpha \sigma} n_{i \bar{\sigma}} \mid d_{j \sigma}^{\dagger}\right\rangle\right\rangle_{\omega}^{r} \\
+ & \sum_{k, \alpha} V_{\alpha i}\left\langle\left\langle d_{i \bar{\sigma}}^{\dagger} c_{k \alpha \bar{\sigma}} d_{i \sigma} \mid d_{j \sigma}^{\dagger}\right\rangle\right\rangle_{\omega}^{r} \\
& -\sum_{k, \alpha} V_{\alpha i}^{*}\left\langle\left\langle c_{k \alpha \bar{\sigma}}^{\dagger} d_{i \bar{\sigma}} d_{i \sigma} \mid d_{j \sigma}^{\dagger}\right\rangle\right\rangle_{\omega}^{r} .
\end{aligned}
$$

To truncate the set of equations for the retarded Green function, we adopt the HFA to the higher-order Green functions on the right side of Eq.(12) and have

$$
\begin{aligned}
\left\langle\left\langle d_{\bar{i} \sigma} n_{i \bar{\sigma}} \mid d_{j \sigma}^{\dagger}\right\rangle\right\rangle_{\omega}^{r} & \cong\left\langle n_{i \bar{\sigma}}\right\rangle\left\langle\left\langle d_{\bar{i} \sigma} \mid d_{j \sigma}^{\dagger}\right\rangle\right\rangle_{\omega}^{r}, \\
\left\langle\left\langle d_{i \sigma} d_{i \bar{\sigma}}^{\dagger} d_{\bar{i} \overline{\bar{\sigma}}} \mid d_{j \sigma}^{\dagger}\right\rangle\right\rangle_{\omega}^{r} \cong\left\langle d_{i \bar{\sigma}}^{\dagger} d_{\bar{i} \overline{\bar{\sigma}}}\right\rangle\left\langle\left\langle d_{i \sigma} \mid d_{j \sigma}^{\dagger}\right\rangle\right\rangle_{\omega}^{r}, & \\
\left\langle\left\langle c_{k \alpha \sigma} n_{i \bar{\sigma}} \mid d_{j \sigma}^{\dagger}\right\rangle\right\rangle_{\omega}^{r} & \cong\left\langle n_{i \bar{\sigma}}\right\rangle\left\langle\left\langle c_{k \alpha \sigma} \mid d_{j \sigma}^{\dagger}\right\rangle\right\rangle_{\omega}^{r}, \\
\left\langle\left\langle d_{i \bar{\sigma}}^{\dagger} c_{k \alpha \bar{\sigma}} d_{i \sigma} \mid d_{j \sigma}^{\dagger}\right\rangle\right\rangle_{\omega}^{r} & \cong\left\langle d_{i \bar{\sigma}}^{\dagger} c_{k \alpha \bar{\sigma}}\right\rangle\left\langle\left\langle d_{i \sigma} \mid d_{j \sigma}^{\dagger}\right\rangle\right\rangle_{\omega}^{r} .
\end{aligned}
$$

Thus obtained retarded Green function is expressed in a compact form as

$$
\mathbf{G}^{r}(\omega)=\left[1-\mathbf{g}^{r}(\omega) \boldsymbol{\Sigma}^{r}\right]^{-1} \mathbf{g}^{r}(\omega),
$$

in which $\boldsymbol{\Sigma}^{r}$ is given by Eqs. (5) and (11), and $\mathbf{g}^{r}(\omega)$ is the Green function for the isolated DQD. It is convenient to express the inverse of $\mathbf{g}^{r}(\omega)$ as

$$
\begin{aligned}
{\left[\mathbf{g}^{r}(\omega)^{-1}\right]_{i i}=} & \frac{\left(\omega-\varepsilon_{i \sigma}\right)\left(\omega-\varepsilon_{i \sigma}-U_{i}\right)}{\omega-\varepsilon_{i \sigma}-U_{i}+U_{i}\left\langle n_{i \bar{\sigma}}\right\rangle} \\
& +\frac{U_{i} t_{c}\left[\left\langle d_{i \bar{\sigma}}^{\dagger} d_{\bar{i} \bar{\sigma}}\right\rangle-\left\langle d_{\bar{i} \bar{\sigma}}^{\dagger} d_{i \bar{\sigma}}\right\rangle\right]}{\omega-\varepsilon_{i \sigma}-U_{i}+U_{i}\left\langle n_{i \bar{\sigma}}\right\rangle}
\end{aligned}
$$

and

$$
\left[\mathbf{g}^{r}(\omega)^{-1}\right]_{i \bar{i}}=t_{c}
$$

Here, the expectation values of $\left\langle n_{1 \bar{\sigma}}\right\rangle$ and $\left\langle d_{i \bar{\sigma}}^{\dagger} d_{\bar{i} \bar{\sigma}}\right\rangle$ can be calculated self-consistently by taking advantage of the definition of the lesser Green function

$$
\left\langle n_{i \bar{\sigma}}\right\rangle=-i \int_{-\infty}^{\infty} \frac{d \omega}{2 \pi} G_{i \bar{\sigma}, i \bar{\sigma}}^{<}(\omega),
$$

and

$$
\left\langle d_{\bar{i} \bar{\sigma}}^{\dagger} d_{\bar{i} \bar{\sigma}}\right\rangle=-i \int_{-\infty}^{\infty} \frac{d \omega}{2 \pi} G_{\bar{i} \bar{\sigma}, i \bar{\sigma}}^{<}(\omega) .
$$

The derivation of advanced Green function $\mathbf{G}^{a}$ follows the same procedure, and its expression is the Hermite conjugate of the retarded Green function.

\section{B. Lesser Green Function}

The lesser Green function is defined by

$$
G_{i \sigma, j \sigma}^{<}(t) \equiv\left\langle\left\langle d_{i \sigma}(t) \mid d_{j \sigma}^{\dagger}\right\rangle\right\rangle^{<} \equiv i\left\langle d_{j \sigma}^{\dagger} d_{i \sigma}(t)\right\rangle .
$$

The equation of motion for the lesser Green function in the Fourier space follows as

$$
\begin{aligned}
& \left(\omega-\varepsilon_{i \sigma}\right)\left\langle\left\langle d_{i \sigma} \mid d_{j \sigma}^{\dagger}\right\rangle\right\rangle_{\omega}^{<}+t_{c}\left\langle\left\langle d_{\bar{i} \sigma} \mid d_{j \sigma}^{\dagger}\right\rangle\right\rangle_{\omega}^{<} \\
= & \sum_{k, \alpha} V_{\alpha i}\left\langle\left\langle c_{k \alpha \sigma} \mid d_{j \sigma}^{\dagger}\right\rangle\right\rangle_{\omega}^{<}+U_{i}\left\langle\left\langle d_{i \sigma} n_{i \bar{\sigma}} \mid d_{j \sigma}^{\dagger}\right\rangle\right\rangle_{\omega}^{<} .
\end{aligned}
$$

The first term on the right hand side of Eq. 19 can be achieved with the analytic continuation ${ }^{30}$ as follows

$$
\begin{aligned}
& \left\langle\left\langle c_{k \alpha \sigma} \mid d_{j \sigma}^{\dagger}\right\rangle\right\rangle_{\omega}^{<}=\sum_{l=1,2} V_{\alpha l}^{*} \\
& \times\left[g_{k \alpha}^{r}(\omega)\left\langle\left\langle d_{l \sigma} \mid d_{j \sigma}^{\dagger}\right\rangle\right\rangle_{\omega}^{<}+g_{k \alpha}^{<}(\omega)\left\langle\left\langle d_{l \sigma} \mid d_{j \sigma}^{\dagger}\right\rangle\right\rangle_{\omega}^{a}\right],
\end{aligned}
$$

where $g_{k \alpha}^{r(a)}$ is the Green function for the noninteracting electrons in the leads,

$$
\begin{aligned}
g_{k \alpha}^{r}(\omega) & =\frac{1}{\omega-\varepsilon_{k \alpha}+i 0^{+}}, \\
g_{k \alpha}^{<}(\omega) & =i f_{\alpha}(\omega) 2 \pi \delta\left(\omega-\varepsilon_{k \alpha}\right) .
\end{aligned}
$$

The equation of motion for the last term on the right hand side of Eq.(19) has almost the same structure with Eq. (12) except for term $\delta_{i j}\left\langle n_{i \bar{\sigma}}\right\rangle$. Under the HartreeFock approximation, by using the expressions similar to Eq.(13), we eventually arrive at

$$
\begin{aligned}
& \left(\omega-\varepsilon_{i \sigma}-U_{i}\right)\left\langle\left\langle d_{i \sigma} n_{i \bar{\sigma}} \mid d_{j \sigma}^{\dagger}\right\rangle\right\rangle_{\omega}^{<} \\
= & -t_{c}\left\langle n_{i \bar{\sigma}}\right\rangle\left\langle\left\langle d_{\bar{i} \sigma} \mid d_{j \sigma}^{\dagger}\right\rangle\right\rangle_{\omega}^{<}-t_{c}\left\langle d_{i \bar{\sigma}}^{\dagger} d_{\bar{i} \bar{\sigma}}\right\rangle\left\langle\left\langle d_{i \sigma} \mid d_{j \sigma}^{\dagger}\right\rangle\right\rangle_{\omega}^{<} \\
+ & t_{c}\left\langle d_{\bar{i} \bar{\sigma}}^{\dagger} d_{i \bar{\sigma}}\right\rangle\left\langle\left\langle d_{i \sigma} \mid d_{j \sigma}^{\dagger}\right\rangle\right\rangle_{\omega}^{<} \\
+ & \sum_{l=1,2}\left\langle n_{i \bar{\sigma}}\right\rangle\left[-\frac{i}{2} \Gamma_{i l}\left\langle\left\langle d_{l \sigma} \mid d_{j \sigma}^{\dagger}\right\rangle\right\rangle_{\omega}^{<}\right. \\
& \left.+i\left(f_{L} \Gamma_{i l}^{L}+f_{R} \Gamma_{i l}^{R}\right)\left\langle\left\langle d_{l \sigma} \mid d_{j \sigma}^{\dagger}\right\rangle\right\rangle_{\omega}^{a}\right] .
\end{aligned}
$$

Inserting Eqs. (20) and (22) into Eq. (19), the expression of $\mathbf{G}^{<}$can be simply cast into

$$
\mathbf{G}^{<}=\mathbf{G}^{r} \boldsymbol{\Sigma}^{<} \mathbf{G}^{a},
$$

where $\boldsymbol{\Sigma}^{<}=i\left(f_{L} \boldsymbol{\Gamma}^{L}+f_{R} \boldsymbol{\Gamma}^{R}\right)$, indicating that, with the second-order HFA, the self energies due to the dotlead coupling are separated from those resulting from Coulomb interaction. 


\section{Current Formula}

Generally, $\mathbf{G}^{r}-\mathbf{G}^{a}=\mathbf{G}^{r}\left(\boldsymbol{\Sigma}^{r}-\boldsymbol{\Sigma}^{a}\right) \mathbf{G}^{a}$. With the discussion above, the Eq.(6) of current is simplified to 28

$$
J=\sum_{\sigma} \frac{e}{h} \int d \omega\left[f_{L}(\omega)-f_{R}(\omega)\right] \operatorname{Tr}\left[\mathbf{G}^{a}(\omega) \boldsymbol{\Gamma}^{R} \mathbf{G}^{r}(\omega) \boldsymbol{\Gamma}^{L}\right],
$$

where $f_{L(R)}=1 /\left\{\exp \left[\left(\omega-\mu \mp \frac{e V}{2}\right) / k_{B} T\right]+1\right\}$, and $V$ is the applied bias voltage. This current expression reduces to a usual Landauer-Büttiker formula for the noninteracting case, implying that, with up to the second order of the HFA, the system can be effectively described by a single-particle picture. In this context, the effect of electrons with spin $\bar{\sigma}$ on the motion of the electron with spin $\sigma$ behaves like a background, and the coherent tunneling process takes place between electrons with the same spin.

\section{NUMERICAL RESULTS AT ZERO TEMPERATURE}

The parameters for our model calculation are taken as follows. For simplicity, we assume $\varepsilon_{1 \sigma}=\varepsilon_{2 \sigma}=\varepsilon_{0}=0$. Throughout this work, we consider the case where the ondot charging energy $U$ is much larger than the interdot coupling $t_{c}$ which is of the order of unity, thus we take $U_{1}=U_{2}=U=4$. As pointed out in Ref 20, in the parallel-coupled geometry, if $\Gamma_{1}^{L}=\Gamma_{2}^{L}$ and $\Gamma_{1}^{R}=\Gamma_{2}^{R}$, the antibonding state could be decoupled entirely from the leads. Our calculation verifies that this situation is retained when the on-dot Coulomb repulsion is taken into account. To avoid it, in the following we only choose two configurations: (1) $\Gamma_{1}^{L}=\Gamma_{2}^{R}>\Gamma_{2}^{L}=\Gamma_{1}^{R}$, and (2) $\Gamma_{1}^{L}=\Gamma_{1}^{R}>\Gamma_{2}^{L}=\Gamma_{2}^{R}$.

It should be pointed out that the numerical results presented in this Section are valid only at temperature above the Kondo temperature, though are calculated at zero temperature, because all the spin flip processes have been neglected as mentioned above.

\section{A. Equilibrium process}

We are particularly interested in how the states in the DQD region are modified by the on-dot Coulomb repulsion, and how this modification influences transport properties. First, let us estimate the eigenstate and eigenenergy of the isolate DQD system.

In the case of only one electron in the DQD system, due to the inter-dot coupling, the DQD states are the linear combination of the states in two dots, thus the formed bonding and antibonding states are associated with energy at $\varepsilon_{0}-t_{c}$ and $\varepsilon_{0}+t_{c}$, respectively. Hence, the one-electron ground state of the DQD is the bonding state with eigenenergy $\varepsilon_{0}-t_{c}$.

When the DQD contains two electrons, 6 possible states in the system include: $|\uparrow\rangle_{1}|\uparrow\rangle_{2},|\downarrow\rangle_{1}|\downarrow\rangle_{2}$, $|\uparrow\rangle_{1}|\downarrow\rangle_{2},|\downarrow\rangle_{1}|\uparrow\rangle_{2},|\uparrow \downarrow\rangle_{1}|0\rangle_{2},|0\rangle_{1}|\uparrow \downarrow\rangle_{2}$. The ground and excited two-electron-states are determined by directly diagonalizing the matrix representation of $\mathrm{H}_{D Q D}$, spanned in the Hilbert space by these 6 states. Thus the two-electron ground state is associated with an energy at $2 \varepsilon_{0}+\frac{1}{2}\left(U-\sqrt{U^{2}+16 t_{c}^{2}}\right) \approx 2 \varepsilon_{0}-4 t_{c}^{2} / U \sim 2 \varepsilon_{0}$. Since the intra-dot Coulomb interaction produces effective charging energy on the bonding and antibonding states, it is expected for the two-electron ground state that the electrons tend to distribute themselves evenly throughout the DQD structure to avoid the charging energy.

Because an extra charging energy has to be consumed to add the third and fourth electron into the system, then the ground states energy is approximately $3 \varepsilon_{0}+U-t_{c}$ and $4 \varepsilon_{0}+2 U$, respectively.

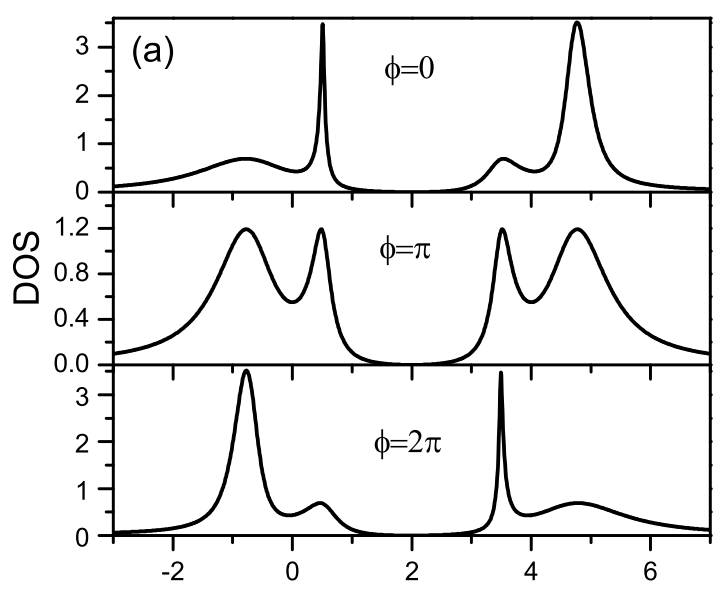

$\omega$

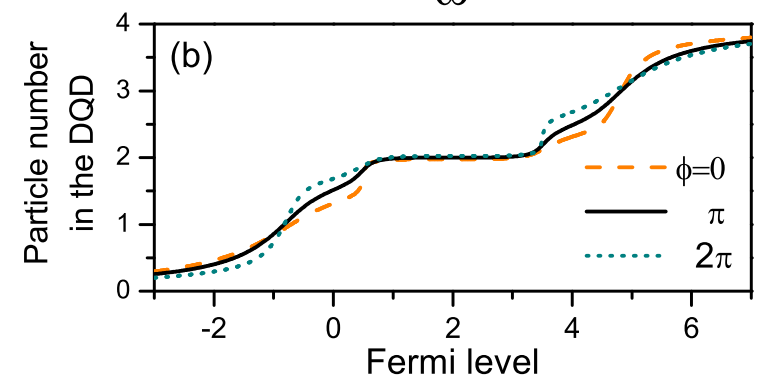

FIG. 2: (Color online) (a)The local density of states and (b) the total particle number in the DQD structure for different magnetic phase parameter $\phi$. A magnetic flux threading into the AB-ring swaps the effective couplings of the DQD states as well as their Coulomb counterparts to the leads. The parameters taken for calculation are: $\varepsilon_{1 \sigma}=\varepsilon_{2 \sigma}=0, t_{c}=1$, $U_{1}=U_{2}=4, \Gamma_{1}^{L}=\Gamma_{2}^{R}=1$ and $\Gamma_{2}^{L}=\Gamma_{1}^{R}=0.15$. Same results are obtained when the dot-lead coupling is changed to $\Gamma_{1}^{L}=\Gamma_{1}^{R}=1$ and $\Gamma_{2}^{L}=\Gamma_{2}^{R}=0.15$.

When an isolate DQD is connected to two leads, the couplings between the DQD levels and two leads result in broadening of the discrete levels and forming bands. The local density of states (DOS), defined as 
the imaginary part of the retarded Green function $\rho_{\sigma}=$ $-\frac{1}{\pi} \sum_{i=1,2} \operatorname{Im} G_{i \sigma, i \sigma}^{r}$, have been calculated for three different magnetic flux $\phi$. Fig. 2(a) reveals that the lineshape of the local DOS critically depends on magnetic flux $\phi$ through the modulation of the effective coupling between the DQD states and leads, which can be tuned by both the dot-lead coupling strength and the total magnetic flux. Fig. 2(a) also shows the band width variation versus the magnetic flux: The broadening of the bonding DQD state is always accompanied by the shrinking of the antibonding DQD state; same for their Coulomb counterparts, though the lineshape of the DQD states and their Coulomb counterparts are somewhat different. It is also noted from Fig. 2(a) that, however, unlike without Coulomb repulsion case ${ }^{23}$ where the total width of the bonding and antibonding bands is an invariant because the self-energy is solely determined by the DQD-leads coupling; with the on-dot Coulomb correlation into consideration, an additional self-energy due to Coulomb repulsion plays a role, then the total band width in general depends on the magnetic flux $\phi$ to some extent.

According to Eq.(24), the differential conductance at equilibrium is defined as $\mathcal{G}(\mu)=\left.\frac{\partial J}{\partial V}\right|_{V \rightarrow 0}$, which at zero temperature reads

$$
\mathcal{G}(\mu)=\sum_{\sigma} \frac{e^{2}}{h} \operatorname{Tr}\left[\mathbf{G}^{a}(\mu) \boldsymbol{\Gamma}^{R} \mathbf{G}^{r}(\mu) \boldsymbol{\Gamma}^{L}\right],
$$

where $\mu$ is the Fermi level. When Fermi level varies, the occupation number of electrons in the DQD region is changed correspondingly. As shown in Fig 2(b), the integer number of electrons confined to the DQD region occurs approximately at the following energies: $\varepsilon_{0}-t_{c}, \varepsilon_{0}+t_{c}, \varepsilon_{0}+U-t_{c}, \varepsilon_{0}+U+t_{c}$

Figs. [3 and 4 show the differential conductance spectra as functions of the Fermi level (or equivalently, of the dot level $\varepsilon_{0}$ ) in two different configurations. The peaks marked with arrows represent the peaks of the Fano-type, whose lineshape is asymmetric compared with the symmetric Lorentzian at the same spectra. Four Lorentzian peaks appear in the conductance spectra when $\phi=\pi$; while there are only two Lorentzian and two Fano peaks for $\phi=0$. The spectra for $\phi=2 \pi$ has a mirror symmetry with that for $\phi=0$.

The peak positions at the DOS and conductance spectra we obtained are in good agreement with those in Refs. 31 and 27, in which two dots coupled in series were considered; however, no Fano effect was observed in the in-series-coupled DQD system. It is then necessary to explain why the Fano interference occurs in the parallelcoupled DQD system, while absents from the in-series DQD system.

As pointed out in our recent article, 23 in general, the lead states couple to the bonding and antibonding levels with different strengths, leading to quite different broadening. If the band width of the strongly-coupled level covers the weakly-coupled band and the phase shift for states in the strongly-coupled channel is negligibly small

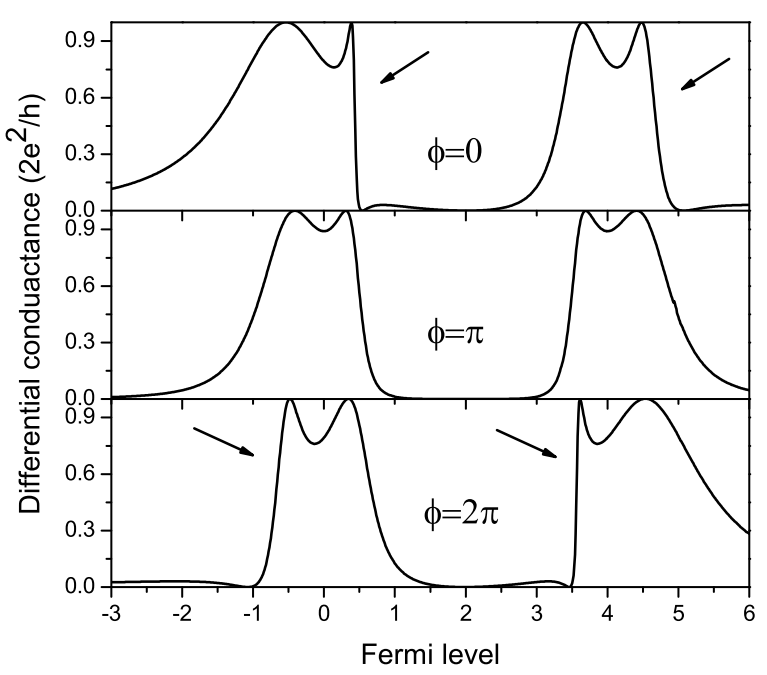

FIG. 3: The differential conductance for configuration 1 as a function of Fermi level. The Fano resonances are marked with arrows. The parameters taken are: $\varepsilon_{1 \sigma}=\varepsilon_{2 \sigma}=0, t_{c}=0.8$, $\Gamma_{1}^{L}=\Gamma_{2}^{R}=1, \Gamma_{1}^{R}=\Gamma_{2}^{L}=0.15$, and $U_{1}=U_{2}=4$.

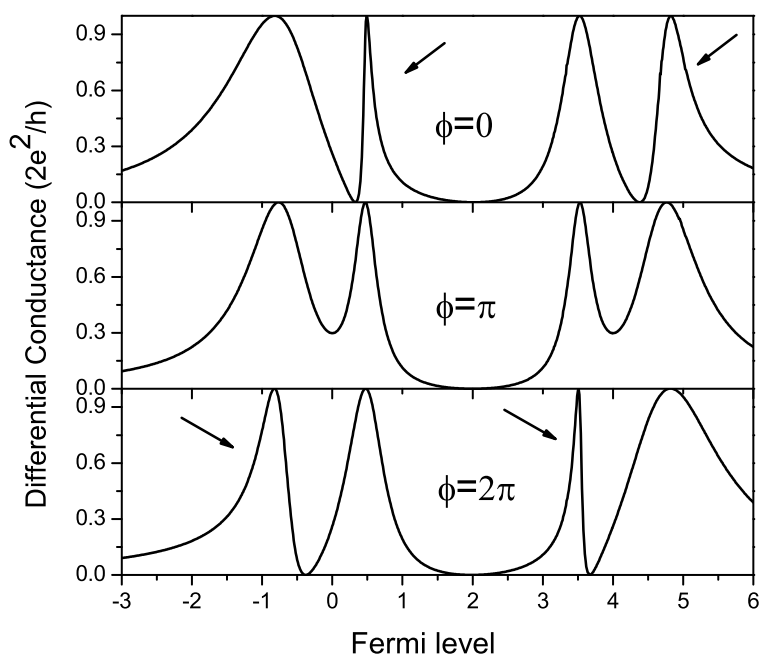

FIG. 4: The differential conductance for configuration 2 as a function of Fermi level. The Fano resonances are marked with arrows. The parameters taken are the same to the figure above except for $t_{c}=1, \Gamma_{1}^{L}=\Gamma_{2}^{L}=1$, and $\Gamma_{1}^{R}=\Gamma_{2}^{R}=0.1$.

when across the weakly-coupled level, then the Fano interference will occur, in which the weakly-coupled channel acts as a Breit-Wigner scatter in the resonant tunneling process while the strongly-coupled channel can be regarded as a non-resonant one. For example, the transmission amplitude of an electron resonantly traversing 
through the a weakly-coupled energy level at $\epsilon_{0}$ can be described $\operatorname{as}^{26}$

$$
t_{R} \frac{\Gamma}{\omega-\epsilon_{0}+i \Gamma},
$$

which implies a phase shift by $\pi$ for the transmission amplitude over an energy range of $\Gamma$ around this level, and $t_{R}$ is a coefficient. On the other hand, the transmission amplitude for states in the strongly-coupled channel can be approximated as $t_{N} e^{i \phi_{N}}$, in which the phase $\phi_{N}$ varies little when across the narrow band centered at $\epsilon_{0}$, the interference between these two channels yields the Fano lineshape around the weakly-coupled level $\epsilon_{0}$ as

$$
\left|t_{R} \frac{\Gamma}{\omega-\varepsilon_{0}+i \Gamma}+t_{N} e^{i \phi_{N}}\right|^{2}=t_{N}^{2} \frac{|\widetilde{\varepsilon}+q|^{2}}{\widetilde{\varepsilon}^{2}+1},
$$

where the detuning $\widetilde{\varepsilon}=\left(\omega-\varepsilon_{0}\right) / \Gamma$, and the asymmetric factor $q=i+t_{R} e^{-i \phi_{N}} / t_{N}$. It should be noted that in the present system, the resonant or non-resonant channel is not fixed, on the contrary, it could be any one of the four channels, as long as the required magnetic flux as well as Fermi level are satisfied.

Notice also that when the dot-lead coupling strength is adjusted such that the configuration 1 (Fig 3 ) is transformed into the configuration 2 (Fig 4), the tail direction of the Fano peak is flipped. The reason behind this behavior is that, compared to configuration 2, an extra flux of $\pi$ threads into the loop in the configuration 1,23 then the Fano lineshape in configuration 1 is just opposite to that in configuration 2. Namely, if two channels interfere with each other constructively in configuration 1 , they will interfere destructively in configuration 2 , and vice versa. Because of different inter-dot coupling $t_{c}$, the splitting between the bonding and antibonding bands in Figs. 3 and 4 is different, resulting in different Fano lineshapes in these two figures.

As for the in-series DQD geometry, the bonding and antibonding states are coupled to the leads with equal strength since one dot has to couple to the left or right leads via the other dot. Though the quantum interference can occur in this geometry as well, one DQD state can not cover or be covered by the spectrum of the other state to give rise to the Fano resonance.

\section{B. Out of Equilibrium}

In the presence of a finite bias voltage $V$, the differential conductance Eq. (24) becomes

$$
\mathcal{G}(\mu)=\sum_{\sigma} \frac{e^{2}}{2 h} \sum_{\alpha=L, R} \operatorname{Tr}\left[\mathbf{G}^{a}\left(\mu_{\alpha}\right) \boldsymbol{\Gamma}^{R} \mathbf{G}^{r}\left(\mu_{\alpha}\right) \boldsymbol{\Gamma}^{L}\right]
$$

where $\mu_{L(R)}=\mu \pm \frac{e V}{2}$, and $\mu=\left(\mu_{L}+\mu_{R}\right) / 2$. Varying $\mu$ is equivalent to adjusting the DQD energy levels reversely. Eq. (28) indicates that the left and right leads contribute to the differential conductance separately. Thus, the
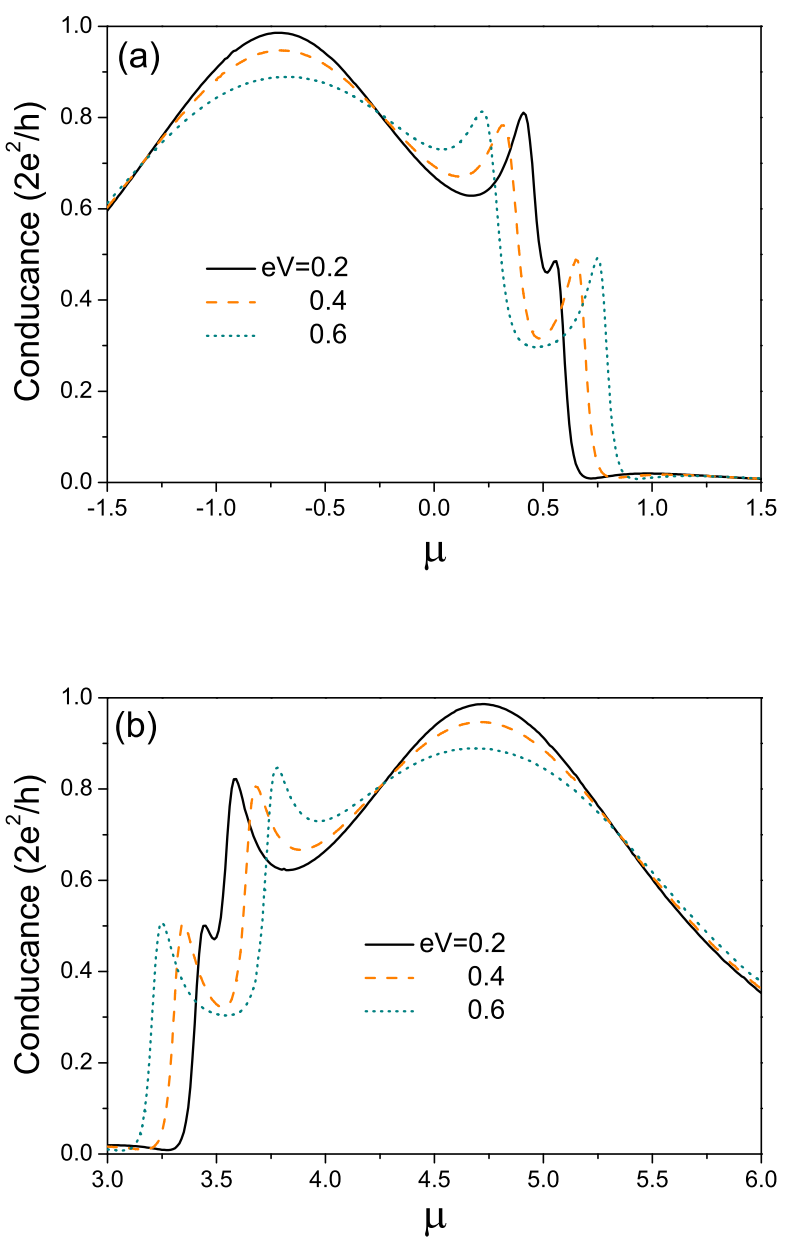

FIG. 5: (Color online) The conductance as functions of the average of the left and right Fermi levels, $\mu$, for different bias voltages in configuration 1 . (a) The resonances at $\varepsilon_{0}-t_{c}$ and $\varepsilon_{0}+t_{c}$ for $\phi=0$. (b) The resonances at $\varepsilon_{0}+U-t_{c}$ and $\varepsilon_{0}+U+t_{c}$ for $\phi=2 \pi$.

Fano peak doublet in the conductance spectra can be expected, when either of two Fermi levels is aligned with the weakly-coupled DQD level under certain circumstance. More specifically, when the system is driven by a bias $V$, if $\mathrm{eV}$ is comparable with or greater than the width of the weakly-coupled level, but less than the width of the strongly-coupled level, the original Fano peak in the conductance spectra may be split. In Fig [ 5 two examples are presented: (a) The conductance spectra around $\varepsilon_{0}-t_{c}$ and $\varepsilon_{0}+t_{c}$ in the absence of magnetic flux, and (b) the spectra around $\varepsilon_{0}+U-t_{c}$ and $\varepsilon_{0}+U+t_{c}$ in the presence of a $2 \pi$ flux. In both cases, three values of $e V$ locate within a region, which is comparable with the width of the weakly-coupled states and less than the width of the strongly-coupled states. It is obvious from the Figure that the splitting of the Fano doublet is proportional to the applied bias, which, together with the 
Lorentzian peak, makes a step-like lineshape in the conductance spectra.

\section{CONCLUSIONS}

In summary, within the Keldysh non-equilibrium Green function formalism and by using the equation of motion method in which the Hatree-Fock approximation is applied to the higher-order Green function, the transport through the parallel-coupled DQD system has been studied with an emphasis put on the effects of the intradot Coulomb correlation on the Fano interference. We have predicted that the Fano effect, as a consequence of quantum interference, could survive in the presence of a finite Coulomb repulsion. Without loss of generality, we select four states corresponding to the bonding and antibonding DQD states and their Coulomb blockade counterparts as the basis for investigation. The coupling between each of these four states and the electrodes broaden the level into a band with different width. The mechanism of the Fano lineshape in conductance spectra is explored. It has been also found that the direction of the asymmetric tail of Fano lineshape can be flipped by tuning the dot-lead couplings. More interestingly, by applying a magnetic flux, linear responses of the four states could be interchanged, leading to a magnetic flux tunable Fano effect. When a suitable bias voltage is applied, the Fano peak doublet and a step-like lineshape may be observed as a result of the splitting of equilibrium Fano resonance.

\section{Acknowledgments}

We would like to acknowledge Hui Zhai, Zuo-zi Chen, Chao-xing Liu, and Zhen-Gang Zhu for helpful discussions. This work is supported by the Natural Science Foundation of China (Grant No. 10374056, 10574076), the MOE of China (Grant No. 2002003089), and the Program of Basic Research Development of China (Grant No. 2001CB610508).
* Electronic address: luhz@castu.tsinghua.edu.cn

$\dagger$ Electronic address: bfzhu@castu.tsinghua.edu.cn

1 U. Fano, Phys. Rev. 124, 1866 (1961).

2 L.P. Kouwenhoven, C.M. Markus, P.L. McEuen, S. Tarucha, R.M. Westervelt, and N.S. Wingreen, in Mesoscopic Electon Transport, edited by L.L. Sohn, L.P. Kouwenhoven, and G. Schön, NATO Advanced Study Institutes, Ser. E, Vol. 345 (Kluwer, Dordrecht, 1997).

3 J. Göres, D. Goldhaber-Gordon, S. Heemeyer, M.A. Kastner, H. Shtrikman, D. Mahalu, and U. Meirav, Phys. Rev. B 62, 2188 (2000).

4 I.G. Zacharia, D. Goldhaber-Gordon, G. Granger, M.A. Kastner, Y.B. Khavin, H. Shtrikman, D. Mahalu, and U. Meirav, Phys. Rev. B 64, 155311 (2001).

5 K. Kobayashi, H. Aikawa, S. Katsumoto, and Y. Iye, Phys. Rev. Lett. 88, 256806 (2002).

6 K. Kobayashi, H. Aikawa, S. Katsumoto, and Y. Iye, Phys. Rev. B 68, 235304 (2003).

7 A.C. Johnson, C.M. Marcus, M.P. Hanson, and A.C. Gossard, Phys. Rev. Lett. 93, 106803 (2004)

8 R.K. Adair, C.K. Bockelman, and R.E. Peterson, Phys. Rev. 76, 308 (1949).

9 U. Fano and J.W. Cooper, Phys. Rev. 137, A1364 (1965).

10 F. Cerdeira, T.A. Fjeldly, and M. Cardona, Phys. Rev. B 8, 4734 (1973).

11 J. Faist, F. Capasso, C. Sirtori, K.W. West, and L.N. Pfeiffer, Nature (London) 390, 589 (1997).

12 A. Yacoby, M. Heiblum, D. Mahalu, and H. Shtrikman, Phys. Rev. Lett. 74, 4047 (1995).

13 R. Schuster, E. Buks, M. Heiblum, D. Mahalu, V. Umansky, and H. Shtrikman, Nature(London) 385, 417 (1997).

14 A.W. Holleitner, C.R. Decker, H. Qin, K. Eberl, and R.H. Blick, Phys. Rev. lett. 87, 256802 (2001).

15 A.W. Holleitner, R.H. Blick, A.K. Hüttel, K. Eberl, and J.P. Kotthaus, Science 297, 70 (2002).

16 A.W. Holleitner, R.H. Blick, and K. Eberl, Appl. Phys.
Lett. 82, 1887 (2003).

17 R.H. Blick, A.K. Hüttel, A.W. Holleitner, E.M. Höhberger, H. Qin, J. Kirschbaum, J. Weber, W. Wegscheider, M. Bichler, K. Eberl, and J.P. Kotthaus, Physica E 16, 76 (2003).

18 J.C. Chen, A.M. Chang, and M.R. Meloch, Phys. Rev. Lett. 92, 176801 (2004).

19 K. Kang and S.Y. Cho, J. Phys.: Condens. Matter 16, 117 (2004).

20 M.L. Ladron de Guevara, F. Claro, and P. A. Orellana, Phys. Rev. B. 67, 195335 (2003).

21 Z.-M. Bai, M.-F. Yang, and Y.-C. Chen, J. Phys.: Condens.Matter 16, 2053 (2004).

22 P.A. Orelana, M.L. Ladrón de Guevara, and F. Claro, Phys. Rev. B 70, 233315 (2004).

23 H. Lu, R. Lü, and B.F. Zhu, Phys. Rev. B. 71, 235320 (2005).

24 D. Loss and D.P. DiVincenzo, Phys. Rev. A 57, 120 (1998).

25 D.P. DiVincenzo, Report No. cond-mat/9612126 also in Ref. 2].

26 A.A. Clerk, X. Waintal, and P.W. Brouwer, Phys. Rev. Lett. 86, 4636 (2001).

27 B.R. Bułka and T. Kostyrko, Phys. Rev. B 70, 205333 (2004).

28 Y. Meir and N.S. Wingreen, Phys. Rev. Lett. 68, 2512 (1992).

29 H. Haug, A.-P. Jauho, "Quantum Kinetics in Transport and Optics of Semiconductors" (Springer-Verlag, 1996).

30 D.C. Langreth, in Linear and Nonlinear Electron Transport in Solids, edited by J.T. Devreese and V.E. Van Daren, Nato ASI, Ser. B, Vol. 17 (Plenum, New York, 1976).

31 G. Klimech, G. Chen, and S. Datta, Phys. Rev. B 502316 (1994). 